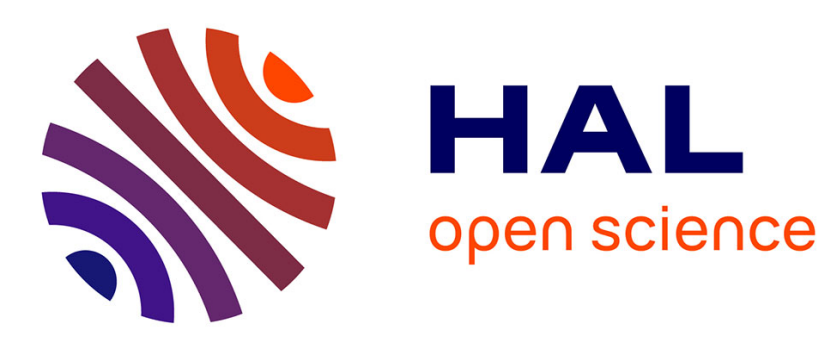

\title{
Low moments of Dirichlet series
}

Hugh L. Montgomery, Gérald Tenenbaum

\section{To cite this version:}

Hugh L. Montgomery, Gérald Tenenbaum. Low moments of Dirichlet series. Acta Mathematica Academiae Scientiarum Hungaricae, 2014, 144 (2), pp.425 - 432. 10.1007/s10474-014-0457-6 . hal01097691

\section{HAL Id: hal-01097691 https://hal.science/hal-01097691}

Submitted on 6 Jan 2015

HAL is a multi-disciplinary open access archive for the deposit and dissemination of scientific research documents, whether they are published or not. The documents may come from teaching and research institutions in France or abroad, or from public or private research centers.
L'archive ouverte pluridisciplinaire HAL, est destinée au dépôt et à la diffusion de documents scientifiques de niveau recherche, publiés ou non, émanant des établissements d'enseignement et de recherche français ou étrangers, des laboratoires publics ou privés. 
Acta Math. Hung. 144,

no. 2 (2014), 425-432

\title{
LOW MOMENTS OF DIRICHLET SERIES
}

\author{
HUGH L. MONTGOMERY AND GÉRALD TENENBAUM
}

Abstract. We determine the maximum possible size of the $q^{\text {th }}$ moment of a Dirichlet series, for $1 \leqslant q \leqslant 2$.

\section{INTRODUCTION}

In order to bound the mean value of multiplicative functions, Halász [5] introduced a majorant principle which (after a little refining) asserts that if $\lambda_{1}, \lambda_{2}, \ldots$ are real numbers, if $\left|a_{n}\right| \leqslant A_{n}$ for all $n$, and $\sum_{n \geqslant 1} A_{n}<\infty$, then

$$
\int_{-T}^{T}\left|\sum_{n \geqslant 1} a_{n} \mathrm{e}^{i \lambda_{n} t}\right|^{2} \mathrm{~d} t \leqslant 3 \int_{-T}^{T}\left|\sum_{n \geqslant 1} A_{n} \mathrm{e}^{i \lambda_{n} t}\right|^{2} \mathrm{~d} t .
$$

For a proof of the principle in this form, see Montgomery [8, §7.3]. In Halász's theory, one needs bounds for integrals of the shape

$$
I(q)=\int_{-1}^{1}\left|\sum_{p} \frac{a_{p} \log p}{p^{\sigma+i t}}\right|^{q} \mathrm{~d} t, \quad J(q)=\int_{-1}^{1}\left|\sum_{n \geqslant 1} \frac{b_{n}}{n^{\sigma+i t}}\right|^{q} \mathrm{~d} t
$$

when $\left|a_{p}\right| \leqslant 1$ for all $p$, and $\left|b_{n}\right| \leqslant 1$ for all $n$. From (1.1) it is immediate that

$$
I(2) \leqslant 3 \int_{-1}^{1}\left|\sum_{n \geqslant 2} \frac{\Lambda(n)}{n^{\sigma+i t}}\right|^{2} \mathrm{~d} t=3 \int_{-1}^{1}\left|\frac{\zeta^{\prime}}{\zeta}(\sigma+i t)\right|^{2} \mathrm{~d} t \ll \int_{-1}^{1} \frac{\mathrm{d} t}{|\sigma+i t-1|^{2}} \ll \frac{1}{\sigma-1}
$$

uniformly for $1<\sigma \leqslant 2$. Similarly, $J(2) \ll 1 /(\sigma-1)$ for $\sigma$ in this range. By applying the majorant principle to the squares of these Dirichlet series we find that $I(4) \ll(\sigma-1)^{-3}$ and $J(4) \ll(\sigma-1)^{-3}$. Hence by Hölder's inequality,

$$
\begin{array}{lll}
I(q) \ll(\sigma-1)^{1-q}, & J(q) \ll(\sigma-1)^{1-q} & (2 \leqslant q \leqslant 4), \\
I(q) \ll(\sigma-1)^{-q / 2}, & J(q) \ll(\sigma-1)^{-q / 2} & (1 \leqslant q \leqslant 2)
\end{array}
$$

uniformly for $1<\sigma \leqslant 2$. The estimate (1.3) is best possible, as we see by taking $a_{p}=1$ for all $p$ and $b_{n}=1$ for all $n$. For purposes of Halász's theory, it would be helpful if (1.3) would hold also when $1<q \leqslant 2$. However, we construct examples that show that the weaker estimate (1.4) is best possible.

Date: November 5, 2014

2010 Mathematics Subject Classification. Primary: 30B50; Secondary: 11Q20, 11N37.

Key words and phrases. Dirichlet series, multiplicative functions. 
Theorem 1.1. Let $I(q)$ and $J(q)$ be defined as in (1.2). There exist numbers $a_{p}$ with $a_{p}= \pm 1$ for all $p$, and $b_{n}$ with $b_{n}= \pm 1$ for all $n$, such that

$$
I(q) \asymp(\sigma-1)^{-q / 2}, \quad J(q) \asymp(\sigma-1)^{-q / 2}
$$

uniformly for $1<\sigma \leqslant 2,1 \leqslant q \leqslant 2$.

This is analogous to the situation for Fourier series. For example, if $\left|b_{n}\right| \leqslant 1$ for $-N \leqslant n \leqslant N$ and $\mathrm{e}(\vartheta)=\mathrm{e}^{2 \pi i \vartheta}$, then

$$
\int_{0}^{1}\left|\sum_{|n| \leqslant N} b_{n} \mathrm{e}(n x)\right|^{q} \mathrm{~d} x \ll N^{q-1}
$$

uniformly for $2 \leqslant q \leqslant 4$, and

$$
\int_{0}^{1}\left|\sum_{|n| \leqslant N} b_{n} \mathrm{e}(n x)\right|^{q} \mathrm{~d} x \ll N^{q / 2}
$$

for $1 \leqslant q \leqslant 2$, but there exists a choice of the $b_{n}$ with $b_{n}= \pm 1$ for all $n$ such that

$$
\int_{0}^{1}\left|\sum_{|n| \leqslant N} b_{n} \mathrm{e}(n x)\right|^{q} \mathrm{~d} x \asymp N^{q / 2}
$$

uniformly for $1 \leqslant q \leqslant 2$. Indeed, we use such $b_{n}$ in our construction.

Antecedents of Halász's majorant principle (1.1) are found in Wiener \& Wintner [15] and in Erdös \& Fuchs [3]. Logan [6] showed that the constant 3 in (1.1) is best-possible.

\section{LemMAS}

We begin with a generalization of a result of H. S. Shapiro [12].

Let the sequence $\left\{r_{n}\right\}_{n=0}^{\infty}$ be defined by the relations $r_{0}=1, r_{2 n}=r_{n}$ and $r_{2 n+1}=(-1)^{n} r_{n}$. The sequence $\left\{r_{n}\right\}_{n=0}^{\infty}$ is the classical Rudin-Shapiro sequence. Suppose that the binary expansion of $n$ is $n=\sum_{j \geqslant 0} e_{j}(n) 2^{j}$ where $e_{j}(n)=0$ or 1 . A well-known alternative definition is

$$
r_{n}=(-1)^{H(n)}, \text { where } H(n):=\sum_{j \geqslant 0} e_{j}(n) e_{j+1}(n) \quad(n \geqslant 0) .
$$

Let $p_{m}(z), q_{m}(z)$ denote polynomials defined recursively by the relations $p_{0}(z)=1$, $q_{0}(z)=1$ and

$$
\begin{aligned}
& p_{m+1}(z)=p_{m}(z)+z^{2^{m}} q_{m}(z) \\
& q_{m+1}(z)=p_{m}(z)-z^{2^{m}} q_{m}(z) .
\end{aligned}
$$

One can easily check that

$$
p_{m}(z)=\sum_{0 \leqslant n \leqslant 2^{m}-1} r_{n} z^{n} \quad(m \geqslant 0) .
$$

The Rudin-Shapiro sequence may be generalized by the so-called paper-folding twist. This amounts to introducing a sequence $\left\{\varepsilon_{m}\right\}_{m=0}^{\infty} \in\{ \pm 1\}^{\mathbb{N}}$ and replacing (2.1) by

$$
\begin{aligned}
& p_{m+1}(z)=p_{m}(z)+\varepsilon_{m} z^{2^{m}} q_{m}(z) \\
& q_{m+1}(z)=p_{m}(z)-\varepsilon_{m} z^{2^{m}} q_{m}(z) .
\end{aligned}
$$


We then obtain

$$
p_{m}(z)=\sum_{0 \leqslant n \leqslant 2^{m}-1} c_{n} z^{n} \quad(m=0,1, \ldots)
$$

with

$$
c_{n}=r_{n} \prod_{j \geqslant 0} \varepsilon_{j}^{e_{j}(n)} \quad(n \geqslant 0),
$$

a formula for which we did not find a reference in the literature and which B. Saffari kindly pointed out to us.

Lemma 2.1. Let the sequence $c_{m}$ be defined as above. Put

$$
P_{M}(\vartheta)=\sum_{0 \leqslant m<M} c_{m} \mathrm{e}(m \vartheta)
$$

Then

$$
\left|P_{M}(\vartheta)\right| \leqslant(2+\sqrt{2}) \sqrt{M}
$$

for all positive integers $M$ and all real $\vartheta$.

Shapiro proved this in the case $c_{m}=r_{m}(m \geqslant 0)$ but never published his work. The coefficients $r_{m}$ were independently discovered by Golay [4]. Rudin [11] published an account of Shapiro's argument in the case $M=2^{k}$, but obtained an inferior constant in the general case. The above lemma is proved in [7, théorème 2].

We note in passing that it follows from the proof of theorem 2 of [7] that, given an arbitrary sequence $\left\{\eta_{j}\right\}_{j=0}^{\infty} \in\{ \pm 1\}^{\mathbb{N}}$, a generalized Rudin-Shapiro sequence may alternatively be written as

$$
c_{m}=(-1)^{v_{m}}
$$

where $v_{m}$ equals 0 or 1 according to whether $\sum_{j \geqslant 0} \eta_{j}\left|e_{j}(m)-e_{j+1}(m)\right|$ belongs to $\{0,1\}$ or to $\{2,3\}$ modulo 4 . In this setting, we recover $r_{m}$ by selecting $\eta_{j}=(-1)^{j}$ $(j \geqslant 0)$. Also, this easily enables retrieving (2.3).

Lemma 2.2. For $|z|<1$, let $f(z)=\sum_{m \geqslant 0} c_{m} z^{m}$ where $c_{m}$ is defined as in (2.3). Then

$$
|f(r \mathrm{e}(\vartheta))| \leqslant \frac{2+\sqrt{2}}{\sqrt{1-r}} .
$$

Numerical studies suggest that, at least in the case $c_{j}=r_{j}(j \geqslant 0)$,

$$
\max _{\vartheta}|f(\operatorname{re}(\vartheta))|=f(r) \text {. }
$$

Moreover, it is easy to show that, in the above circumstance, $f(r) \sqrt{1-r}$ does not tend to a limit as $r \rightarrow 1^{-}$. Indeed, it is proved in Brillhart, Erdős and Morton [1] that

$$
\sum_{m<n} r_{m}=\sqrt{n} G\left(\frac{\log n}{\log 4}\right) \quad(n \geqslant 0)
$$

where $G$ is 1-periodic and continuous. Moreover, Dumont and Thomas [2] showed that $G$ is nowhere differentiable and Tenenbaum [13] obtained the oscillation result

$$
G(x+h)-G(x)=\Omega(\sqrt{h}) \quad(h \geqslant 0)
$$

for any given real number $x$. 
By partial summation it is readily derived from (2.7) that $f(r) \sqrt{1-r}$ oscillates as $r \rightarrow 1-$ : indeed, as $y \rightarrow \infty$,

$$
2^{y} f\left(\exp \left(-4^{-y}\right)\right)
$$

tends to a 1-periodic, nowhere differentiable function of $y$.

It is noteworthy that

$$
\begin{aligned}
f(z) & =f\left(z^{2}\right)+f\left(-z^{2}\right), \\
f(-z) & =f\left(z^{2}\right)-f\left(-z^{2}\right) .
\end{aligned}
$$

Kumiko Nishioka [10] showed that $f(z)$ and $f(-z)$ are algebraically independent, and then used these recurrences and Mahler's method to show that if $\alpha$ is algebraic with $0<|\alpha|<1$, then $f(\alpha)$ and $f(-\alpha)$ are algebraically independent.

Proof. Clearly

$$
\frac{f(r \mathrm{e}(\vartheta))}{1-r}=\sum_{m \geqslant 0} P_{m+1}(\vartheta) r^{m} .
$$

Hence by Lemma 2.1 and the triangle inequality it follows that

$$
\left|\frac{f(r \mathrm{e}(\vartheta))}{1-r}\right| \leqslant(2+\sqrt{2}) \sum_{m \geqslant 0} \sqrt{m+1} r^{m} .
$$

But

$$
\sqrt{m+1} \leqslant\left(\begin{array}{c}
m+1 / 2 \\
m
\end{array}\right)
$$

for all non-negative integers $m$. Hence the right hand side of $(2.10)$ is

$$
\leqslant(2+\sqrt{2}) \sum_{m \geqslant 0}\left(\begin{array}{c}
m+1 / 2 \\
m
\end{array}\right) r^{m}=\frac{2+\sqrt{2}}{(1-r)^{3 / 2}},
$$

which gives the stated result.

To prove $(2.11)$, let $a_{m}=\left(\begin{array}{c}m+1 / 2 \\ m\end{array}\right) / \sqrt{m+1}$. To show that $a_{m} \geqslant 1$, it suffices to note that $a_{0}=1$, and to show that the $a_{m}$ are increasing. As to this latter point, we observe that

$$
\frac{a_{m}}{a_{m-1}}=\frac{m+1 / 2}{\sqrt{m(m+1)}}=\frac{2 m+1}{\sqrt{(2 m+1)^{2}-1}}>1 .
$$

Lemma 2.3. Let $f$ be defined as in Lemma 2.2. For each $r, 0<r<1$, there is a measurable set $A_{r} \subseteq \mathbb{T}$ with Lebesgue measure $\lambda\left(A_{r}\right) \geqslant 1 / 50$, such that

$$
|f(r \mathrm{e}(\vartheta))| \geqslant \frac{1}{2 \sqrt{1-r}}
$$

for all $\vartheta \in A_{r}$.

Proof. Let $B_{r}=\mathbb{T} \backslash A_{r}$ be the complementary set of those $\vartheta$ on which $|f|$ is small; precisely $|f(r e(\vartheta))|<1 /(2 \sqrt{1-r})$. By Parseval's identity we know that

$$
\int_{0}^{1}|f(r \mathrm{e}(\vartheta))|^{2} \mathrm{~d} \vartheta=\sum_{m \geqslant 0} r^{2 m}=\frac{1}{1-r^{2}}>\frac{1}{2(1-r)} .
$$


By Lemma 2.2, the left hand side above equals

$$
\int_{A_{r}}|f(r \mathrm{e}(\vartheta))|^{2} \mathrm{~d} \vartheta+\int_{B_{r}}|f(r \mathrm{e}(\vartheta))|^{2} \mathrm{~d} \vartheta \leqslant \frac{(2+\sqrt{2})^{2}}{1-r} \lambda\left(A_{r}\right)+\frac{1-\lambda\left(A_{r}\right)}{4(1-r)} .
$$

On combining this with $(2.13)$, we find that

$$
\frac{1}{4} \leqslant\left((2+\sqrt{2})^{2}-\frac{1}{4}\right) \lambda\left(A_{r}\right),
$$

which gives the stated result.

Lemma 2.4. Write $s=\sigma+i t$. Then

$$
\sum_{n \leqslant x} \frac{\Lambda(n)}{n^{s}}=-\frac{\zeta^{\prime}}{\zeta}(s)+\frac{x^{1-s}}{1-s}+O\left(\frac{x^{1-\sigma}}{\exp (\sqrt{\log x})}\right)
$$

for $x \geqslant 2,1<\sigma \leqslant 2,-1 \leqslant t \leqslant 1$.

This is included in equation (III.5.72) of [14] and is proved by Perron's summation formula (see Montgomery-Vaughan [9, Theorem 5.2] or Tenenbaum [14, corollary II.2.4]) appealing to the classical zero-free region and estimates for $\zeta^{\prime}(s) / \zeta(s)$ in the zero-free region.

Lemma 2.5. For $x \geqslant 2,1<\sigma \leqslant 2$, and $-1 \leqslant t \leqslant 1$, we have

$$
\sum_{n \leqslant x} \frac{1}{n^{s}}=\zeta(s)+\frac{x^{1-s}}{1-s}+O\left(\frac{1}{x^{\sigma}}\right) .
$$

This is immediate by partial summation; see Montgomery-Vaughan [9, Theorem 1.12] or Tenenbaum [14, theorem II.3.5] for the details.

\section{Proof of the Theorem}

In view of the upper bounds of (1.4), it suffices to establish lower bounds. For $I(q)$ we let $c_{m}$ be defined as in (2.3), and take $a_{p}:=c_{m}$ for $\mathrm{e}^{\pi m}<p<\mathrm{e}^{\pi(m+1)}$. Then, for $s=\sigma+i t, 1<\sigma \leqslant 2,|t| \leqslant 1$,

$$
\begin{aligned}
\sum_{p} \frac{a_{p} \log p}{p^{s}} & =\sum_{m \geqslant 0} c_{m} \sum_{\mathrm{e}^{\pi m}<p<\mathrm{e}^{\pi(m+1)}} \frac{\log p}{p^{s}} \\
& =\sum_{m \geqslant 0} c_{m}\left(\sum_{\mathrm{e}^{\pi m}<n<\mathrm{e}^{\pi(m+1)}} \frac{\Lambda(n)}{n^{s}}+O\left(\mathrm{e}^{\pi m(1-2 \sigma)}\right)\right) .
\end{aligned}
$$

By Lemma 2.4 this is

$$
=\sum_{m \geqslant 0} c_{m}\left(\frac{\mathrm{e}^{\pi(m+1)(1-s)}}{1-s}-\frac{\mathrm{e}^{\pi m(1-s)}}{1-s}\right)+O\left(\sum_{m \geqslant 0} \frac{\mathrm{e}^{\pi m(1-\sigma)}}{\exp (c \sqrt{m})}\right)+O(1) .
$$

Here the first error term is also $O(1)$, uniformly for $\sigma \geqslant 1$. The main term is

$$
F(s) f\left(e^{\pi(1-s)}\right)
$$

where $f$ is defined in Lemma 2.2 , and

$$
F(s)=\frac{e^{\pi(1-s)}-1}{1-s} .
$$

The zeros of this entire function are the numbers $1+2 i m$ where $m$ runs over non-zero integers. Thus $|F(s)|$ is bounded away from 0 uniformly on the rectangle $1 \leqslant \sigma \leqslant 2$, 
$-1 \leqslant t \leqslant 1$. With a little computation one can in fact show that the minimum of $|F(s)|$ in this rectangle is $|F(2 \pm i)| \approx 0.73766$. By Lemma 2.3 it follows that if $\sigma$ is fixed, $1<\sigma \leqslant 2$, then

$$
\left|\sum_{p} \frac{a_{p} \log p}{p^{\sigma+i t}}\right| \gg \frac{1}{\sqrt{1-\mathrm{e}^{\pi(\sigma-1)}}} \asymp \frac{1}{\sqrt{\sigma-1}}
$$

when $t / 2 \in A_{\mathrm{e}^{\pi(\sigma-1)}}$, i.e. on a subset of $-1 \leqslant t \leqslant 1$ of measure $>1 / 25$. Hence $I(q) \gg(\sigma-1)^{-q / 2}$.

The proof for $J(q)$ is the same, except that now the passage from $\log p$ to $\Lambda(n)$ in (3.1) is unnecessary, and instead of Lemma 2.4 we use Lemma 2.5, in which the error term is smaller.

\section{REFERENCES}

[1] J. Brillhart, P. Erdős \& P. Morton, On sums of Rudin-Shapiro coefficients II, Pacific J. Math. 107 (1983), 39-69.

[2] J.-M. Dumont \& A. Thomas, Systèmes de numération et fonctions fractales relatifs aux substitutions, Theoret. Comput. Sci. 65 (1989), 153-169.

[3] P. Erdős \& W. H. J. Fuchs, On a problem in additive number theory, $J$. London Math. Soc. 31 (1956), 67-73.

[4] M. J. E. Golay, Multi-slit spectroscopy, J. Opt. Soc. Amer. 39 (1949), 437444.

[5] G. Halász, Über die Mittelwerte multiplikativer zahlentheoretischer Funktionen, Acta Math. Acad. Sci. Hungar. 19 (1968), 365-403.

[6] B. F. Logan, An interference problem for exponentials, Michigan Math. J. 35 (1988), 369-393.

[7] M. Mendès France \& G. Tenenbaum, Dimension des courbes planes, papiers pliés, et suites de Rudin-Shapiro, Bull. Soc. Math. France 109 (1981), 207215.

[8] H. L. Montgomery, Ten Lectures on the Interface between Analytic Number Theory and Harmonic Analysis, CBMS No. 84, Amer. Math. Soc. (Providence), 1994, xiii+220 pp.

[9] H. L. Montgomery \& R. C. Vaughan, Multiplicative Number Theory I: Classical Theory, Cambridge University Press (Cambridge), 2007, xvii+552 pp.

[10] K. Nishioka, New approach in Mahler's method, J. Reine Angew. Math. 407 (1990), 202-219.

[11] W. Rudin, Some theorems on Fourier coefficients, Proc. Amer. Math. Soc. 10 (1959), 855-859.

[12] H. S. Shapiro, Extremal problems for polynomials and power series, M.S. Thesis, Massachusetts Institute of Technology, 1951.

[13] G. Tenenbaum, Sur la non-dérivabilité de fonctions périodiques associées à certaines fonctions sommatoires, in: R.L. Graham \& J. Nešetřil (eds), The Mathematics of Paul Erdös, Algorithms and combinatorics 13 (Springer Verlag, 1997), 117-128.

[14] G. Tenenbaum, Introduction à la théorie analytique et probabiliste des nombres, troisième édition, coll. Échelles, Belin, 2008, 592 pp.

[15] N. Wiener \& A. Wintner, On a local $L^{2}$-variant of Ikehara's theorem, Rev. Math. Cuyana 2 (1956), 53-59; Norbert Wiener: Collected Works, vol. II, MIT Press, Cambridge, 1979, pp. 758-764; 788-790. 
Department of Mathematics, University of Michigan, Ann Arbor, Mi 48109-1043 E-mail address: hlm@umich.edu

Institut Élie Cartan, Faculté des Sciences, Université de Lorraine, B.P. 70239, 54506 Vandeuvre-lès-Nancy Cedex, France

E-mail address: gerald.tenenbaum@univ-lorraine.fr 